Commun. math. Phys. 29, 43-47 (1973)

(C) by Springer-Verlag 1973

\title{
Tidal Accelerations in General Relativity
}

\author{
John Stewart and Martin Walker \\ Max-Planck-Institut für Physik und Astrophysik \\ München, Germany
}

Received July 3, 1972

\begin{abstract}
We derive the kinematical magnification of tidal accelerations which occurs for test bodies in highly relativistic orbits preferentially aligned with respect to repeated principal null rays of algebraically special spacetimes. Some examples of astrophysical importance are discussed. A general expression for computing tidal accelerations in the Newman-Penrose formalism is given.
\end{abstract}

\section{Introduction}

A problem of current interest in relativistic astrophysics is the behaviour of test bodies near black holes. This behaviour is likely to be most extreme when the speed $v$ of the body approaches the local speed of light $c$. One question which arises in such a situation is whether tidal accelerations experienced by the body can become large enough to tear it apart. On the basis of previous work of Pirani [1] it might be thought that the magnitude of the tidal accelerations $T=O\left(\gamma^{2}\right)$ as $\gamma \rightarrow \infty$, where $\gamma=\left(1-v^{2} c^{-2}\right)^{-1 / 2}$. In this case, a body whose velocity approached the local light velocity arbitrarily closely would presumably be torn apart. It is clearly of importance to determine under what conditions such a kinematical magnification of tidal accelerations does or does not take place.

In the next section, we present a theorem which gives sufficient conditions for the kinematical magnification mentioned above not to take place. The theorem applies only to situations in which the gravitational field (Weyl tensor) is algebraically special, but since there is strong evidence [2] to suggest that the exterior of a black hole can be described by one of the Kerr family of spacetimes (which are all of a particular algebraically special type), this restriction is not as severe as it may seem. In the third section we discuss briefly two cases in which the theorem does apply and one in which it does not, all of which are of potential astrophysical interest [3]. Finally in the fourth section we give an expression for the tidal acceleration in a completely general spacetime which may include both nongravitational fields and a cosmological term. 
We are aware of other work on tidal accelerations experienced by bodies moving in circular orbits in Kerr's spacetime [4], but we believe our approach warrants attention because of its simplicity, generality, and lucidity. The simplicity is in large measure due to our use of a Newman-Penrose (NP) [5] null tetrad adapted to the repeated principal null direction $(R P N D)$ of the field.

\section{The General Result}

The models which have been proposed to describe the gravitational field exterior to a black hole are all of the algebraically special type, that is to say, they all admit (at least) one $R P N D$ of the Weyl tensor. We have computed the tidal accelerations experienced by a test body, the tangent vector to whose orbits approaches arbitrarily closely (in a sense shortly to be made explicit) to the null vector corresponding to a $R P N D$.

Theorem. Let $\left(\mathscr{M}, g_{a b}\right)$ be a spacetime whose Weyl tensor is algebraically special with $R P N D l^{a}$. Include $l^{a}$ in an $N P$ tetrad $l^{a}, n^{a}, m^{a}, \bar{m}^{a}$, and let $\tau$ be a timelike geodesic whose unit tangent vector $t^{a}$ is arbitrarily close to $l^{a}$ in the following sense: if

$$
t^{a}=A l^{a}+B n^{a}+\bar{C} m^{a}+C \bar{m}^{a},
$$

then there exists a real, positive $\varepsilon$ such that

$$
A=O\left(\varepsilon^{-1}\right), \quad B=O(\varepsilon)
$$

as $\varepsilon \rightarrow 0$. If $\zeta^{a}$ and $\eta^{a}$ are unit spacelike vectors orthogonal to $t^{a}$, then a typical component of the tidal acceleration

has magnitude

$$
T=C_{a b c d} \eta^{a} t^{b} \zeta^{c} t^{d}
$$

$$
\begin{array}{ll}
T=O\left(\varepsilon^{-1}\right) & \text { for type } I I(\{211\}) \\
T=O(1) & \text { for type } D(\{22\}) \\
T=O(\varepsilon) & \text { for type } I I I(\{31\}) \\
T=O\left(\varepsilon^{2}\right) & \text { for type } N(\{4\}) .
\end{array}
$$

Proof. Since $t^{a} t_{a}=1$, we have $2(A B-C \bar{C})=1$, so that $C=O(1)$ follows from $A=O\left(\varepsilon^{-1}\right), B=O(\varepsilon)$. If we expand

$$
\zeta^{a}=X l^{a}+Y n^{a}+\bar{Z} m^{a}+Z \bar{m}^{a},
$$

then $\zeta^{a} \zeta_{a}=-1$ implies $2(X Y-Z \bar{Z})=-1$, while $t^{a} \zeta_{a}=0$ implies $A X+B Y=C \bar{Z}+\bar{C} Z$, from which we may deduce $X=O\left(\varepsilon^{-1}\right)$, 
$Y=O(\varepsilon), Z=O(1)$. If

$$
\eta^{a}=X^{\prime} l^{a}+Y^{\prime} n^{a}+\bar{Z}^{\prime} m^{a}+Z^{\prime} \bar{m}^{a},
$$

then a similar result obtains also for the primed quantities. Substituting these expressions into the general formula for $T$ given in the last section, and recalling that $n^{a}, m^{a}, \bar{m}^{a}$ can be chosen so that only certain tetrad components of the Weyl tensor survive for each algebraically special type $^{1}$, we obtain the desired result.

\section{Applications}

We illustrate the above theorem with three cases involving trajectories near black holes. Consider first a radially infalling body in Schwarzschild's spacetime. Since the inward directed radial null direction is one $R P N D$ (the other being the outward directed radial null direction), the body's trajectory can fulfill the conditions of the theorem for type $\{22\}$, which then states the well known result [6] that a body falling radially through the Schwarzschild horizon experiences tidal accelerations $T=O\left(\mathrm{~m}^{2}\right)$, ( $m$ being the mass), with no peculiar kinematical effects.

A less trivial example occurs in an extreme ( $a=m$, where $a$ is the angular momentum parameter) Kerr spacetime, where we consider the stable timelike circular orbits, in the equatorial plane, arbitrarily close to the event horizon at $r=m$. Here one must be a little careful, since the repeated principal null vector of interest in the usual notation is the vector $n^{a}$, which generates the horizon [7]. (The vector $l^{a}$, in the usual notation, points inward across the horizon.) For a field of type $\{22\}$, however, the only nonvanishing tetrad component of the curvature is $\Psi_{2}$, provided both $l^{a}$ and $n^{a}$ are chosen as $R P N D$ 's, so the theorem is symmetric in $l^{a}$ and $n^{a}$, and we obtain the result that no kinematical magnification occurs in this case: the tidal accelerations remain finite right up to the horizon.

Finally we consider an example in Schwarzschild's spacetime, somewhat analogous to the Kerr example, where the theorem does not apply. There is a family of unstable circular timelike geodesics approaching the circular null orbits at $r=3 \mathrm{~m}$. Considering an orbit

${ }^{1}$ For each type, the tetrad may be chosen as follows:

$$
\begin{aligned}
&\{211\}: \Psi_{0}=\Psi_{1}=\Psi_{4}=0 . \\
&\{22\}: \Psi_{0}=\Psi_{1}=\Psi_{3}=\Psi_{4}=0 . \\
&\{31\}: \Psi_{0}=\Psi_{1}=\Psi_{2}=\Psi_{4}=0 . \\
&\{4\}: \Psi_{0}=\Psi_{1}=\Psi_{2}=\Psi_{3}=0 .
\end{aligned}
$$


at $r=3 m(1+\varepsilon)$, we find

$$
A=1 / 6 \varepsilon^{-1 / 2}+O(1), \quad B=\varepsilon^{-1 / 2}+O(1), \quad C=3^{-3 / 2} \varepsilon^{-1 / 2}+O(1)
$$

as $\varepsilon \rightarrow 0$. In this case the tangent to the orbit is not preferentially oriented with respect to the curvature (the $R P N D$ 's being radial), and we find $T=O\left(\varepsilon^{-1}\right)$. Tidal accelerations are indeed kinematically magnified, as one might have been lead to expect from the Pirani result [1].

\section{General Tidal Acceleration Formula}

To fix signs and conventions, we define the Riemann tensor by

$$
2 \nabla_{[a} \nabla_{b]} t_{c}=R_{a b c d} t^{d},
$$

where $\nabla_{a}$ denotes covariant derivative. If $t^{c} \nabla_{c}=D, D t^{a}=0$, so that $t^{a}$ is geodetic, and $\zeta^{a}$ is a vector orthogonal to and commuting with $t^{a}$, then the Jacobi or geodesic deviation equation is

$$
D^{2} \zeta^{a}=R_{b c d}^{a} t^{b} \zeta^{c} t^{d} .
$$

Here $\alpha \zeta^{a}, \alpha \ll 1$, can be thought of as a vector connecting a pair of infinitesimally neighbouring geodesics; $\alpha D^{2} \zeta^{a}$ is then the relative acceleration of the two. Let $\eta^{a}$ be any vector orthogonal to $t^{a}$. Then the component of the relative or tidal acceleration corresponding to $\eta^{a}$ is

$$
T=\eta_{a} D^{2} \zeta^{a}=R_{a b c d} \eta^{a} t^{b} \zeta^{c} t^{d} .
$$

Let $l^{a}, n^{a}, m^{a}, \bar{m}^{a}$ be a null tetrad, and the $\Psi^{\prime} s, \Phi^{\prime} s$ and $\Lambda$ the curvature quantities defined by $N P$ [5]. Then if

$$
\begin{aligned}
& t^{a}=A l^{a}+B n^{a}+\bar{C} m^{a}+C \bar{m}^{a} \\
& \zeta^{a}=X l^{a}+Y n^{a}+\bar{Z} m^{a}+Z \bar{m}^{a} \\
& \eta^{a}=X^{\prime} l^{a}+Y^{\prime} n^{a}+\overline{Z^{\prime}} m^{a}+Z^{\prime} \bar{m}^{a},
\end{aligned}
$$

a straightforward, if lengthy, calculation gives

$$
\begin{aligned}
T= & \Psi_{0}(\bar{C} X-A \bar{Z})\left(\bar{C} X^{\prime}-A \bar{Z}^{\prime}\right) \\
+ & +\Psi_{1}(\bar{C} X-A \bar{Z})\left(B X^{\prime}-A Y^{\prime}+\bar{C} Z^{\prime}-C \overline{Z^{\prime}}\right) \\
+ & +\left(\bar{C} X^{\prime}-A \bar{Z}^{\prime}\right)(B X-A Y+\bar{C} Z-C \bar{Z}) \\
+\Psi_{2}(B Z-C Y)\left(\bar{C} X^{\prime}-A \bar{Z}^{\prime}\right) & \\
& +(B X-A Y+\bar{C} Z-C \bar{Z})\left(B X^{\prime}-A Y^{\prime}+\bar{C} Z^{\prime}-C \bar{Z}^{\prime}\right) \\
& +\left(B Z^{\prime}-C Y^{\prime}\right)(\bar{C} X-A \bar{Z}) \\
+ & \left(B X^{\prime}-A Y^{\prime}+\bar{C} Z^{\prime}-C \overline{Z^{\prime}}\right) \\
& +\left(B Z^{\prime}-C Y^{\prime}\right)(B X-A Y+\bar{C} Z-C \bar{Z})
\end{aligned}
$$




$$
\begin{aligned}
& +\Psi_{4}(B Z-C Y)\left(B Z^{\prime}-C Y^{\prime}\right) \\
& +2 \Lambda(A B-C \bar{C})\left(X^{\prime} Y+X Y^{\prime}-Z^{\prime} \bar{Z}-Z \bar{Z}^{\prime}\right) \\
& +\Phi_{00}\left(C X^{\prime}-A Z^{\prime}\right)(\bar{C} X-A \bar{Z}) \\
& +\left[\Phi_{01}(\bar{C} X-A \bar{Z})+\Phi_{21}(B Z-C Y)\right]\left(B X^{\prime}-\bar{C} Z^{\prime}+C \bar{Z}^{\prime}-A Y^{\prime}\right) \\
& +\left[\Phi_{10}\left(C X^{\prime}-A Z^{\prime}\right)+\Phi_{12}\left(B \bar{Z}^{\prime}-\bar{C} Y^{\prime}\right)\right](B X-C \bar{Z}+\bar{C} Z-A Y) \\
& +\Phi_{02}\left(B \overline{Z^{\prime}}-\bar{C} Y^{\prime}\right)(\bar{C} X-A \bar{Z}) \\
& +\Phi_{11}(B X-C \bar{Z}+\bar{C} Z-A Y)\left(B X^{\prime}-\bar{C} Z^{\prime}+C \bar{Z}^{\prime}-A Y^{\prime}\right) \\
& +\Phi_{20}(B Z-C Y)\left(C X^{\prime}-A Z^{\prime}\right) \\
& +\Phi_{22}\left(B \bar{Z}^{\prime}-\bar{C} Y^{\prime}\right)(B Z-C Y) \\
& + \text { complex conjugate. }
\end{aligned}
$$

Despite the cumbersome appearance of this expression, it is remarkably easy to use in the (by now numerous) cases for which appropriate tetrads and $N P$ quantities are known.

Acknowledgements. We wish to thank J. Ehlers, P. Kafka, and D. W. Sciama for discussions.

\section{References}

1. Pirani, F.A.E.: Proc. Roy. Soc. Edinburgh A 252, 96 (1959).

2. Carter, B.: Phys. Rev. Letters 26, 331 (1971).

3. Misner, C.: Phys. Rev. Letters 28, 994 (1972).

4. Fishbone, L.: Private communication from C. Misner.

5. Newman, E. T., Penrose, R.: J. Math. Phys. 3, 566 (1962).

6. Finley, J.D.: J. Math. Phys. 12, 32 (1971).

7. Kinnersley, W.: J. Math. Phys. 10, 1195 (1969).

John Stewart

Martin Walker

Max-Planck-Institut für Physik

und Astrophysik

D-8000 München 23

Föhringer Ring 6

Federal Republic of Germany 
